\title{
Psychiatry Investigation : The Next Step Forward
}

Psychiatry Investigation(PI) has been published biannually since August 2004 as an official English journal of the Korean Neuropsychiatric Association(KNPA) for the purpose of dealing with unique regional psychiatric issues. Under the leadership of the KNPA president Prof. Yong Sik Kim, Prof. Byoung-Hoon Oh became the founding Editor-in-Chief of the Journal. Launching on a new English journal was not an easy task and rather very challenging one. The editorial team under the pioneering leadership of Prof. Oh invested significant personal time, efforts and resources into PI.

After 5 years of publication, PI became indexed in Science Citation Index-Expanded (SCIE) in April 2009 thanks to profound contribution of the second Editor-in-Chief, Prof. Chan-Hyung Kim and his editorial board members. It was not only a success of editorial team but also a success of all the KNPA members.

From 2008, PI has been publishing quarterly and many papers with high quality from all over the world have been submitted for publication. Currently, PI is under evaluation to be indexed in PubMed Central by the National Institute of Health(NIH), USA. If PI can be indexed in PubMed Central, the papers in PI can be searched in PubMed browser of NIH and researchers all over the world can access the full-text papers of our journal for free. Being a PubMed Central journal would be another success of PI and KNPA.

However, our challenge of PI does not end here, it is only a beginning. Our next goals remain to get the journal indexed in the SCI core and Medline. To achieve these goals, increasing citation from researchers is crucial. The citations received by PI have increased over the last few years, but it is still not enough. More citations are needed to reach our goals.

For these purposes, we should publish highly qualified papers in the Journal. I have plans to increase current rejection rate of around $15 \%$ up to $30 \%$ in the near future. Considering rapidly increasing numbers of paper submission, it would be possible. Highly qualified review articles and original articles are invited and very welcomed to the Journal, which would contribute on increasing citations of PI. On the other hand, keeping the uniqueness of scope in psychiatric field is also important to be a renowned world class journal. PI will continue to focus on the cross cultural psychiatry and ethnic issues in psychiatry in Asian countries as well as general psychiatry and neurosciences, which is our unique scope comparing to other journals.

Our new editorial team will do our best to achieve these goals for the further development of PI, and we look forward to informing you more about our plan and hope in the actual forthcoming issues. 